\title{
Incidence and prevalence of Systemic Sclerosis (SSc) in Poland - differences between rural and urban regions
}

\author{
Krzysztof Kanecki', Paweł Goryński², Patryk Tarka', Waldemar Wierzba ${ }^{3}$, Piotr Tyszko ${ }^{4}$ \\ ${ }^{1}$ Department of Social Medicine and Public Health, Medical University of Warsaw, Poland \\ ${ }^{2}$ National Institute of Public Health - National Institute of Hygiene, Poland \\ ${ }^{3}$ University of Humanities and Economics in Lodz, Poland \\ ${ }^{4}$ Institute of Rural Health, Lublin, Poland
}

Kanecki K, Goryński P,Tarka P, WierzbaW, Tyszko P. Incidence and prevalence of Systemic Sclerosis (SSc) in Poland - differences between rural and urban regions. Ann Agric Environ Med. 2017; 24(2): 240-244. doi: 10.5604/12321966.1233570

\begin{abstract}
Introduction. Systemic sclerosis (SSc) is a rare and potentially severe connective tissue disease, characterized by skin fibrosis and involvement of internal organs. Because of its rarity and heterogeneous clinical presentation, reliable epidemiological studies on SSc have been particularly difficult to carry out.

Objectives. The purpose of this study was to present SSc epidemiology among hospitalized patients in Poland.

Materials and method. The analysis was based on population-based administrative data, taken from a Polish hospital morbidity study carried out by the National Institute of Public Health between January 2008 - December 2012.

Results. Analyzed data covered 9,049 hospitalization records. The final sample comprised 3,653 patients with first-time hospitalizations for SSc. The average age of the sample was 53 years (SD 16.2; range 0-91 years); 84\% of patients were female and $16 \%$ male. Based on hospitalization registers, the average SSc incidence was estimated to be 1.9/100,000 per year and peak age of incidence was 55 years. The point prevalence was estimated to be $9.4 / 100,000$ at the end of 2012 . SSc was more common in females, with F:M ratio ranging from 6.2:1-4.6:1 depending on the year. Analysis of hospitalization trends revealed that overall numbers of SSc hospitalizations increased, while first-time hospitalizations decreased between 2008-2012. Clusters of higher incidence were observed in more rural regions vs. urban regions.

Conclusion. Estimated incidence of SSc in Poland was comparable to reported incidence in other European countries. Analysis of demographic factors and reports of clusters of higher incidence may suggest the existence of environmental risk factors for the development of SSc. These observations may require further research.
\end{abstract}

Key words

epidemiology, rare diseases, hospitalization

\section{INTRODUCTION}

Systemic sclerosis (SSc) is a rare and potentially severe connective tissue disease, characterized by skin fibrosis, involvement of internal organs, an unpredictable course, high mortality and resistance to therapy. The complexity and severity of SSc is a growing burden on health-care systems.

Scleroderma refers to a heterogeneous group of autoimmune fibrosing disorders. Currently accepted disease subtypes include scleroderma with morphea (localized scleroderma), limited cutaneous systemic sclerosis (ISSc), diffuse cutaneous systemic sclerosis (dcSSc), and systemic sclerosis sine scleroderma (ssSSc). The classification of SSc into ISSc, dcSSc, and ssSSc subsets is the one that most closely reflects the natural history of the disease, as each of these subtypes reflect clear clinical differences [1].

Although morphea and systemic sclerosis result in activation of similar inflammatory and fibrotic pathways, important differences in signaling pathways and gene signatures indicate they are likely biologically distinct processes. Morphea can cause significant morbidity but it

Address for correspondence: Krzysztof Kanecki,Department of Health Care, Medical University of Warsaw, 02-097 Warsaw, Oczki 3, Poland.

E-mail:kanecki@mp.pl

Received: 22 February 2016; accepted: 13 July 2016; first published on February 2017 is not associated with higher mortality in comparison to the general population. Systemic sclerosis has the highest disease-specific mortality of all autoimmune connective tissue diseases.

The etiology of SSc is unknown. The remarkable complexity of its pathogenesis suggests that no single gene or environmental trigger, by itself, is likely to be responsible for the developments of SSc. Genetic factors clearly influence disease susceptibility as well as patterns of disease expression. The occurrence of SSc-like disease in response to certain environmental agents and the possible relationship of SSc to environmental exposures, suggest that certain triggers lead to variable disease expression in genetically-susceptible hosts. SSc has been associated with occupational exposures to crystalline silica, white spirit, aromatic solvents, chlorinated solvents, trichlorethylene, ketones and welding fumes [2]. Associations of these agents with SSc appear to be more marked in those with high cumulative exposure, and the association between SSc and occupational exposures may be variable according to gender [2].

Occupational exposure to crystalline silica/solvents is correlated with more severe forms of SSc, characterized by diffuse cutaneous involvement, interstitial lung disease, general microangiopathy (digital ulcers and myocardial dysfunction), and association with cancer) [3]. 
Prevalence and incidence. The estimated prevalence of SSc in a large cohort in the United States for the period 19891991 was 240 per million adults (95\% CI 213-274) with an annual incidence of 19.3 new cases per million adults per year (95\% CI 12.4-30.2), gender- and race-specific prevalence estimates were significantly higher for women than for men, and for blacks than for whites [4]. Other population studies suggest that SSc occurs more frequently in the United States than other regions, such as continental Europe, the United Kingdom, and in some areas in Asia [5]. SSc prevalence in Quebec, Canada was higher among older individuals, particularly in urban women (161.2 cases per 100,000 ; $95 \%$ CI 148.6-175.0), and lowest in young men in rural areas, (2.8 cases per 100,000; 95\% CI 1.4-4.8) [6]. The prevalence of SSc in Alberta, Canada, was 58 cases per 100,000 females (95\% CI 51-65) and 10 cases per 100,000 males (95\% CI 7-14), with a trend toward higher SSc prevalence in rural residents [7]. In the multi-ethnic country of France, the prevalence of SSc (per 100,000 adults) was 15.8 (95\% CI: 12.9-18.7) and respective prevalences for European and non-European residents of France were 14 per 100,000 (95\% CI: 11.2-17) and 21 per 100,000 (95\% CI: 12.8-29.3), which suggests that there may be an influence of ethnic origin on the pathogenesis of SSc [8]. A study conducted in southeast Norway on a sample of $2,707,012$ individuals (56\% of the total Norwegian population) used the ACR and/or the Medsger and LeRoy SSc criteria to determine a point prevalence of 9.9/100,000 $(95 \%$ CI 8.8, 11.2) in 1999-2009. The estimated prevalences of 1SSc, lcSSc and dcSSc subcategories were 1.3/100,000 (95\% CI $0.9-1.8$ ), 6.9/100,000 (95\% CI 5.8-7.8) and 1.8/100,000 (95\% CI 1.4-2.5), respectively [9].

The adult prevalence in 1998-2010 and annual incidence in 2006-2010 of SSc in the Skåne region in Sweden were 30.5 and 1.9 per 100,000 inhabitants, and a majority (82\%) of the prevalent cases had the lcSSc subtype [10].

In a systematic literature review analyzing articles published from 1969-2006, the yearly international prevalence of SSc increased from $0.7 / 100,000-48.9 / 100,000$, and the yearly incidence increased from $0.06 / 100,000-12.2 / 100,000$ [11]. In this review, an unusually high prevalence of SSc was reported in some regions. Ontario, Canada, Rome, Italy, and areas near airports in London, England, reported rates of SSc that were 3, 5, and 1,000 times greater than expected, respectively. Thus, there appears to be spatio-temporal clustering of SSc.

Incidence may be more difficult to estimate than prevalence due to differences in physician awareness of the disease and inconsistency in the reliability of ascertainment methods. The overall incidence of SSc among United States adults is approximately 20 per million per year [5]. In northwestern Spain, the age- and gender-adjusted average annual incidence over the 19-year study period was 2.3 (95\% CI, 1.6-2.5) per 100,000 population aged 15 years and older. The incidence was higher in women (3.5 per 100,000 [95\% CI, 2.3-3.9]) than men (1.0 per 100,000 [95\% CI, 0.5-1.4]); $\mathrm{p}<0.001$ ) [12].

Risk factors. Evidence from multiple sources indicates that SSc does not occur randomly in the population; there are particular groups who are at greater risk. While SSc may occur at any age, it mainly affects adults. SSc is more common in females and among individuals of African origin. Reports of sporadic clusters of higher prevalence suggest that there may be environmental risk factors. Exposure to silica was first suggested as a risk factor for SSc in 1917, and is officially recognized in France for purposes of workers' compensation. Exposure to silica dust appears to be an environmental trigger, but this only accounts for a small proportion of male cases [13]. Occupational exposures associated with SSc development include crystalline silica, white spirit, aromatic solvents, chlorinated solvents, trichloroethylene, ketones and welding fumes [2]. Solvent exposure has been associated with SSc in several rigorous case-control studies. Data on the relationship between SSc and other suspected toxic agents (epoxy resins, vibrations, welding fumes) is currently insufficient to draw causal inferences [14]. The severity of SSc (determined by the extent of diffuse cutaneous lesions, pulmonary involvement, and immunological profile) is probably associated with occupational exposure [14].

Survival in SSc. Compared to the general population, lifespan among patients with SSc is significantly reduced [12]. Lung involvement and sclerodermal renal crisis were found to be independently associated with reduced survival [15]. Cardiac involvement, diffuse cutaneous SSc, and hypertension are associated with worse survival [16]. Other studies have confirmed the presence of diffuse cutaneous forms and renal involvement as independent risk factors for SSc-related mortality [17]. Post-diagnosis survival has improved over the past few decades, with current mean survival approximately 12 years after diagnosis [5]. Age at disease onset has been associated with differences in clinical presentation and disease course [18]. Older patients with SSc are at greater risk for pulmonary hypertension, renal impairment, cardiac disease, and muscle weakness [19].

Gastrointestinal tract (GIT) involvement commonly affects patients with SSc, causing complications like malabsorption, weight loss and severe malnutrition [20]. In a recent study, it was reported that the Total GIT Score provides greater discrimination between mild, moderate, and severe self-rated GIT involvement than individual scales [21].

Juvenile or childhood onset systemic sclerosis is rare. Patients with juvenile onset SSc more frequently have an overlap syndrome with PM-DM, higher frequency of skeletal muscle involvement, serum anti-PM-Scl and anti-U1RNP antibody, fatal cardiac disease, and improved survival compared with adult onset SSc cases [22]. In patients with juvenile onset disease, the prevalence of lung fibrosis and scleroderma renal crisis was low; however, the rate of cardiac involvement was higher than that in adult patients [23].

\section{OBJECTIVES}

The purpose of this study was to present SSc epidemiology among hospitalized patients in Poland.

\section{MATERIALS AND METHOD}

The analysis was based on population-based administrative data, collected between January 2008 - December 2012. Analyzed data covered 9,049 hospitalization records, 3,653 of which reflected first time hospitalizations for SSc. Data were collected by the National Institute of Public Health as part of the Polish hospital morbidity study. Data on all inpatients discharged alive or dead from all hospitals, excluding psychiatric and military hospitals, are obligatorily 
sent to the Institute, usually on a monthly basis. The database contains information about each hospitalization, including ICD-10 codes of the admitting diagnosis and comorbidities, dates of admission and discharge, birth date, gender, and place of residence. The database does not contain patients' names. Demographic data for the general Polish population was obtained from The Central Statistical Office in Poland [24]. Two analytic samples were considered in the current study: 1) all hospitalizations for SSc during the study period, and 2) all hospitalization records of patients with a first-time hospitalization for SSc. SSc as potentially severe connective tissue disease, characterized by skin fibrosis and involvement of internal organs, often requires hospitalization and therefore, analysis of hospitalization cases may provide a good estimate of the SSc incidence and prevalence in Poland.

Statistical analysis. Statistical analyses were performed using Statistica Software (StatSoft, Inc., 2011). Descriptive statistics assessed included means, medians and ranges for continuous variables, and absolute numbers and proportions for categorical data.

\section{RESULTS}

Between 2008-2012, there were 9,049 hospitalizations of patients admitted with SSc. Among these, 3,653 SSc patients were hospitalized for the first time. The mean age of the sample was 53 years (median 55; SD 16.2; range: $0-91$ ). The study sample was comprised of $584(16 \%)$ male and 3,065 (84\%) female patients. The gender of four patients was recorded as 'undetermined'. Based on hospitalization records and census data, the yearly incidence of SSc was estimated to be $1.9 / 100,000$, with peak incidence occurring at the age of 55 years and the point prevalence to be $9.4 / 100,000$ at the end of 2012.

Over the course of the study period, the overall number of hospitalizations increased, while the number of first-time hospitalizations (used as a proxy of incident SSc), decreased. Details about first time and all hospitalizations of SSc patients in every analyzed year are presented in Tables 1 and 2 .

Among SSc patients with first time hospitalizations, 65\% resided in more urban areas, $33 \%$ resided in more rural areas, and no information about residence was available for $2 \%$ of patients. For all SSc hospitalizations combined, the urban vs. rural breakdown was similar.

The sample of all hospitalizations consisted of 1,568 (17.3\%) male patients and 7,477 (82.6\%) female patients, with 4 patients of undetermined gender. The mean age for all hospitalizations was 53.2 years (SD 14.6; median: 55; range: 1-91). Among overall SSc hospitalizations, more than half $(53.4 \% ; n=4834)$ were related to a progressive form of SSc, followed by ISSc $(4.3 \% ; n=389)$. Other hospitalizations were related to other forms of SSc.

During the study period, 62 SSc patient died while hospitalized. The most common causes of death were cardiac failure $(n=28)$, respiratory failure $(n=16)$, and SSc $(n=6)$. Hospitalizations for SSc were mainly in the departments of rheumatology (64\%), dermatology (19.5\%), internal medicine (6.2\%), allergology (1.8\%), and paediatrics (1.1\%). There was no seasonality in overall and first-time SSc hospitalizations.
Table 1. All hospitalizations in 2008-2012

\begin{tabular}{lccccc}
\hline & 2008 & 2009 & 2010 & 2011 & 2012 \\
\hline Progressive SSc & 823 & 999 & 920 & 1068 & 1024 \\
\hline LSSC syndrome & 51 & 78 & 70 & 92 & 98 \\
\hline SSc - others & 680 & 717 & 744 & 867 & 818 \\
\hline All & 1554 & 1794 & 1734 & 2027 & 1940 \\
\hline F/M - All hospitalizations & 5.6 & 5.5 & 4.6 & 4.4 & 4.2
\end{tabular}

LSSC - limited cutaneous form of systemic sclerosis (Icssc). The acronym 'LSSC' refers to the 5 main features: calcinosis, Raynaud's phenomenon, esophageal dysmotility, sclerodactyly and telangiectasia. F/M: female - male ratio

Table 2. First hospitalizations for SSc in 2008-2012

\begin{tabular}{lccccc}
\hline & 2008 & 2009 & 2010 & 2011 & 2012 \\
\hline Progressive SSC & 428 & 303 & 221 & 199 & 190 \\
\hline LSSC syndrome & 51 & 49 & 44 & 35 & 34 \\
\hline SSc - others & 521 & 447 & 365 & 401 & 365 \\
\hline All & 1000 & 799 & 630 & 635 & 589 \\
\hline F/M -First hospitalizations & 6.2 & 5.8 & 5.1 & 4.3 & 4.6 \\
\hline
\end{tabular}

F/M: female - male ratio

There were several regional clusters of higher first time hospitalizations. Distribution of first time hospitalizations in various regions of Poland per 100,000 people is shown in Figure 1. Higher rates of first-time hospitalizations were seen in the southeastern and eastern regions of Poland, which are predominantly agricultural.

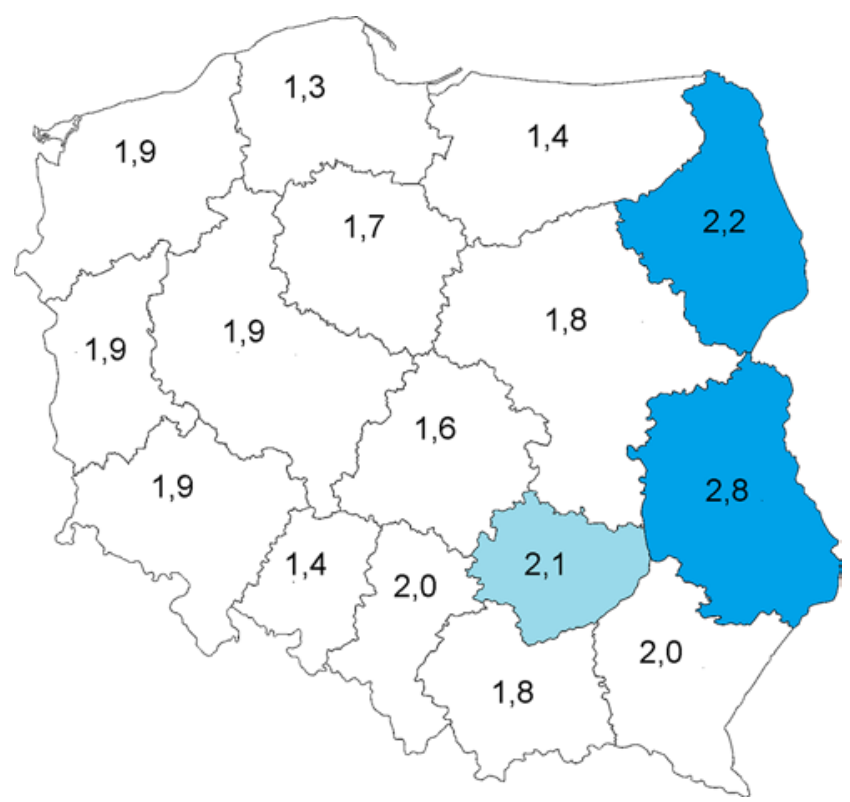

Figure 1. First hospitalizations of SSc patients in the Polish provinces (per 100,000 per year)

SSc incidence in this study was more common in females, with the average F:M ratio presented in Tables 1 and 2. $\mathrm{SSc}$ was more common in females, with F:M ratio from 6.2:1 -.6:1, depending on the year. The F:M ratio decreased over time, and was more marked among patients with progressive SSc. The age distribution in patients with first time hospitalizations is presented in Figure 2. The median age at first time hospitalization with SSC diagnosis was 53 years (median:55, range: $0-91$ years). 


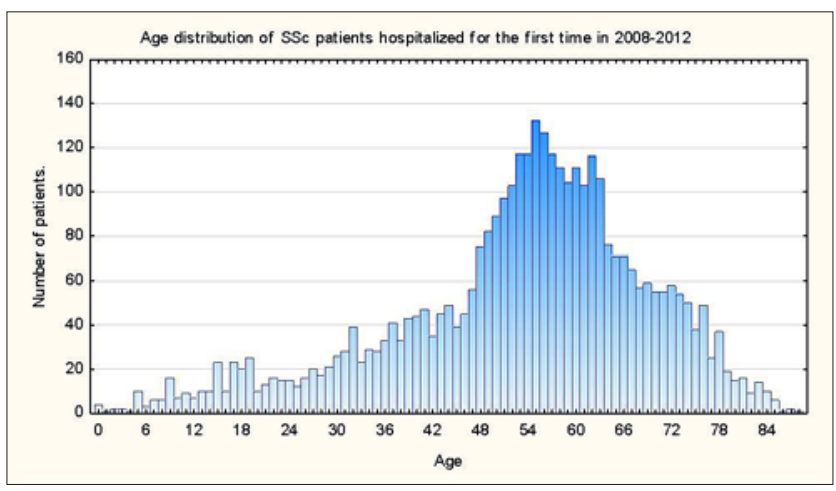

Figure 2. Age distribution of SSc patients, 2008-2012.

\section{DISCUSSION}

Because of its rarity and heterogeneous clinical presentation, reliable epidemiological studies on SSc have been particularly difficult to carry out. The epidemiological data on SSc epidemiology in many European countries have been presented in many articles, but no similar data based on a Polish patient population have been published.

Systemic scleroderma, and especially its progressive character, almost always requires diagnosis and treatment in a hospital. The presented study used reported first-time hospitalization as a proxy for incident scleroderma in Poland. The overall annual incidence rate of SSc in the population of the Poland was estimated at 1.9 per 100,000 per year, an incidence comparable with data from the USA [5] and European countries [10, 12].

In the current study, a gradual increase in all hospitalizations of SSc patients was noted, especially for patients diagnosed with progressive SSc. The observed gradual increase may be related to the presence of an unknown factor, such as infectious, allergic, or demographic changes. In northwestern Spain, the overall incidence rates of SSc also increased over the length of an epidemiologic study [12]. Additionally, the observation in the current of a gradual decrease in the female: male ratio over time, and higher rates of first-time hospitalizations that were seen in the southeastern and eastern regions of Poland, may support the concept of an environmental, infectious, or other trigger in a geneticallysusceptible population.

In the presented study, there was a decrease over time in the number of patients hospitalized for the first time. This may indicate that the burden of diagnosis, treatment and care of patients with SSC has shifted to outpatient care. On the other hand, the decrease could be related to the presence of other or unknown infectious, allergic or environmental factors, or the organization of care in Poland. For all hospital admissions of patients with SSc, including those not directly associated with scleroderma, Poland is among the countries with the highest number of hospitalizations [25]. These problems, however, may require further study.

In this study, the peak incidence of SSc occurred at the age of 55 years, and the female to male ratio ranged from 6.2:1-4.6:1. In Norway, the mean age at onset was 47 years and the female:male ratio was 3.8:1. In a Spanish sample, the incidence of SSc was higher in individuals aged 45 years or older, and the mean age at the time of disease diagnosis was $59.8+/-13.3$ years].
There are a few limitations in this study. An important limitation is that the study relied on hospital discharge records, without information from outpatients. This inaccuracy eventually may result in an underestimate of the true incidence rate. The fact that a case first appeared in the nationwide morbidity registry with a diagnosis of SSc does not necessarily mean that the diagnosis was newly-made. However, the long-term period of analysis seems to greatly improve the ability to assess the SSc incidence.

\section{CONCLUSIONS}

The estimated SSc incidence in Poland was 1.9/100 000 per year, and was comparable to data from other European countries. The point prevalence was estimated to be 9.4 $/ 100,000$ at the end of 2012. Between 2008-2012, there were 3,653 hospitalizations of patients admitted with SSc for the first time. Analysis of demographic factors and reports of clusters of higher incidence may suggest the existence of environmental risk factors for the development of SSc. These observations may require further research.

\section{REFERENCES}

1. Simeon-Aznar CP, Fonollosa-Pla V, Tolosa-Vilella C, EspinosaGarriga G, Ramos-Casals M, Campillo-Grau M, et al. Registry of the Spanish network for systemic sclerosis: clinical pattern according to cutaneous subsets and immunological status. Semin Arthritis Rheum. 2012; 41(6): 789-800, http://dx.doi.org/10.1016/j. semarthrit.2011.10.004

2. Marie I, Gehanno JF, Bubenheim M, Duval-Modeste AB, Joly P, Dominique $S$, et al. Prospective study to evaluate the association between systemic sclerosis and occupational exposure and review of the literature. Autoimmun Rev. 2014; 13(2): 151-6, http://dx.doi. org/10.1016/j.autrev.2013.10.002.

3. Marie I, Menard JF, Duval-Modeste AB, Joly P, Dominique S, Bravard $\mathrm{P}$, et al. Association of occupational exposure with features of systemic sclerosis. J Am Acad Dermatol. 2015; 72(3): 456-64, http://dx.doi. org/10.1016/j.jaad.2014.11.027.

4. Mayes MD, Lacey JV, Jr., Beebe-Dimmer J, Gillespie BW, Cooper B, Laing TJ, et al. Prevalence, incidence, survival, and disease characteristics of systemic sclerosis in a large US population. Arthritis Rheum. 2003; 48(8): 2246-55, http://dx.doi.org/ 10.1002/art.11073

5. Mayes MD. Scleroderma epidemiology. Rheum Dis Clin North Am. 2003; 29(2): 239-54.

6. Bernatsky S, Joseph L, Pineau CA, Belisle P, Hudson M, Clarke AE. Scleroderma prevalence: demographic variations in a populationbased sample. Arthritis Rheum. 2009; 61(3): 400-4, http://dx.doi. org/10.1002/art.24339.

7. Barnabe C, Joseph L, Belisle P, Labrecque J, Edworthy S, Barr SG, et al. Prevalence of systemic lupus erythematosus and systemic sclerosis in the First Nations population of Alberta, Canada. Arthritis Care Res (Hoboken). 2012; 64(1): 138-43, http://dx.doi.org/10.1002/acr.20656.

8. Le Guern V, Mahr A, Mouthon L, Jeanneret D, Carzon M, Guillevin L. Prevalence of systemic sclerosis in a French multi-ethnic county. Rheumatology (Oxford). 2004; 43(9): 1129-37, http://dx.doi.org/10.1093/ rheumatology/keh253.

9. Hoffmann-Vold AM, Midtvedt O, Molberg O, Garen T, Gran JT. Prevalence of systemic sclerosis in south-east Norway. Rheumatology (Oxford). 2012; 51(9): 1600-5, http://dx.doi.org/10.1093/rheumatology/ kes076.

10. Andréasson K, Saxne T, Bergknut C, Hesselstrand R, Englund M. Prevalence and incidence of systemic sclerosis in southern Sweden: population-based data with case ascertainment using the 1980 ARA criteria and the proposed ACR-EULAR classification criteria. Ann Rheum Dis. 2014; 73(10): 1788-92, http://dx.doi.org/10.1136/ annrheumdis-2013-203618.

11. Chifflot H, Fautrel B, Sordet C, Chatelus E, Sibilia J. Incidence and prevalence of systemic sclerosis: a systematic literature review. Semin 
Arthritis Rheum. 2008; 37(4): 223-35, http://dx.doi.org/10.1016/j. semarthrit.2007.05.003.

12. Arias-Nunez MC, Llorca J, Vazquez-Rodriguez TR, Gomez-Acebo I, Miranda-Filloy JA, Martin J, et al. Systemic sclerosis in northwestern Spain: a 19-year epidemiologic study. Medicine (Baltimore). 2008; 87(5): 272-80, http://dx.doi.org/10.1097/MD.0b013e318189372f.

13. Barnes J, Mayes MD. Epidemiology of systemic sclerosis: incidence, prevalence, survival, risk factors, malignancy, and environmental triggers. Curr Opin Rheumatol. 2012; 24(2): 165-70, http://dx.doi. org/10.1097/BOR.0b013e32834ff2e8.

14. Magnant J, Diot E. [Systemic sclerosis: epidemiology and environmental factors]. Presse Med. 2006; 35(12 Pt 2): 1894-901. French

15. Simeón CP, Armadans L, Fonollosa V, Solans R, Selva A, Villar M, et al. Mortality and prognostic factors in Spanish patients with systemic sclerosis. Rheumatol. 2003; 42: 71-5.

16. Al-Dhaher FF, Pope JE, Ouimet JM. Determinants of morbidity and mortality of systemic sclerosis in Canada. Semin Arthritis Rheum. 2010; 39: 269-77.

17. Ranque B, Mouthon L. Geoepidemiology of systemic sclerosis. Autoimmun Rev. 2010; 9(5): A311-8, http://dx.doi.org/10.1016/j. autrev.2009.11.003.

18. Alba MA, Velasco C, Simeon CP, Fonollosa V, Trapiella L, Egurbide MV et al. Early- versus late-onset systemic sclerosis: differences in clinical presentation and outcome in 1037 patients. Medicine (Baltimore). 2014; 93(2): 73-81, http://dx.doi.org/10.1097/md.0000000000000018.

19. Manno RL, Wigley FM, Gelber AC, Hummers LK. Late-age onset systemic sclerosis. J Rheumatol. 2011; 38(7): 1317-25, http://dx.doi. org/10.3899/jrheum.100956.

20. Nagaraja V, McMahan ZH, Getzug T, Khanna D. Management of gastrointestinal involvement in scleroderma. Curr Treatm Opt Rheumatol. 2015; 1: 82-105.

21. Khanna D, Hays RD, Maranian P, Seibold JR, Impens A, Mayes MD, et al. Reliability and validity of the University of California, Los Angeles Scleroderma Clinical Trial Consortium Gastrointestinal Tract Instrument. Arthritis Rheum. 2009; 61: 1257-63.

22. Scalapino K, Arkachaisri T, Lucas M, Fertig N, Helfrich DJ, Londino AV Jr, et al. Childhood onset systemic sclerosis: classification, clinical and serologic features, and survival in comparison with adult onset disease. J Rheumatol. 2006; 33(5): 1004-13, http://dx.doi.org/.

23. Aoyama K, Nagai Y, Endo Y, Ishikawa O. Juvenile systemic sclerosis: report of three cases and review of Japanese published work. J Dermatol. 2007; 34(9): 658-61, http://dx.doi.org/10.1111/j.1346-8138.2007.00350.x.

24. Central Statistical Office of Poland. [cited 1014 Oct 13]. Available from: http://stat.gov.pl

25. World Health Organization Regional Office for Europe. [cited 2014 Oct 27]. Available from: www.euro.who.int. 